welcher sie sich sofort nach dem Ausschlüpfen aus der Mutterzelle äusserst lebhaft vorwärts bewegen, häufig zeigen sie eine schnelle, rotirende Bewegung um sich selbst, wobei der rothe Punkt den Mittelpunkt bildet. Nach kurzer Zeit (bei kultivirten Individuen nach ungefähr 24 Stunden\} kopuliren sie sich, indem sie sich zugleich an die nächsten Gegenstände festsetzen, und zwar legen sie sich mit ihren entgegengesetzten Enden aneinander, ziehen die Cilien ein, umgeben sich mit einer Membran, aus welcher nach ebenfalls nicht langer Zeit das Produkt, die Zygozoospore, schlüpft, die sofort zur neuen Pflanze auswächst. Einen Unterschied zwischen männlichen und weiblichen Schwärmsporen habe ich bis jetzt nicht beobachten können, obwohl unter den Schwärmern sich schlankere, mehr zugespitzte und breitere mit mehr abgerundeter Spitze zeigen.

Was die systematische Stellung anbelangt, so gehört diese Alge sicher in die Nähe von Aphanochaete A. Br. und Bolbocoleon Pringsh. etc. oder ist dem einen oder dem andern Genus beizuordnen, was erst bei genauerer Kenntniss der übrigen Arten entschieden werden kann.
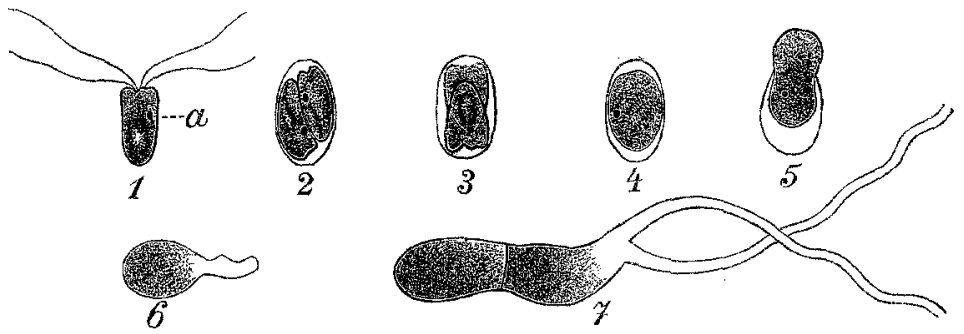

Frklärung der Zeichnung. Vergrösserung 480.

1. Eine Schwärmspore, bei a der rothe Punkt.

2. Zwei Schwärmsporen in Copulation.

3. Dieselben. Ansicht von der Seite.

4. Produkt derselben, die Zygozoospore.

כ. Das Austreten der Zygozoospore aus der Hüllmembran.

6. Das Auswachsen zur neuen Pflanze.

7. Weitere Entwicklung.

\title{
Ueber einige Gräser Spaniens und Portugals.
}

Von Prof. Fd. Hackel.

Die folgenden Beobachtungen sammelte ich auf einer Reise in den obgenannten Ländern, die ich im Sommer 1876 in Gesellschaft meines werthen Freundes Moritz Winkler, den Lesern dieser Zeitschrift durch seine "Reiseerinnerungen aus Spanien (Jahrgang 1875) bekannt, unternahm. Auf Willkomm's trefflicher Bearbeitung der Grä- 
ser im Prodromus Florae hispanicae fussend, sollen sie bei kritischen Arten seine kurzgefassten Diagnosen ergänzen, eine schärfere Unterscheidung schwieriger Arten versuchen, ferner auf manche interessante Formen bekannter Spezies aufmerksam machen und endlich eine Anzahl neuer wichtigerer Standorte angeben.

1. Lygeum Spartum L. Bei Carthagena sammelte ich eine var. barbatum, welche unterhalb der Einfügungsstelle der Spatha ein Haarbüschel besitzt. Auch fand ich daselbst durch Brand missbildete Exemplare mit 4-5 Scheiden, deren jede die bekannten zwei verwachsenen Aehrchen im Winkel trug. Erwähnenswerth scheint mir noch, dass die Ligula dieses Grases von zwei Gefässbündeln durchzogen ist, sowie, dass an der Spitze der Spatha stets eine kleine Ligula und eine verkümmerte Lamina angedeutet ist, die in Ausnahmsfällen auch zu voller Entwicklung gelangen.

Das Gras dürfte auch in ganzen Südösten Spaniens auf wüsten, steinigen Hügeln verbreitel sein, ich sammelte es bei Callosa, Carthagena und Almeria.

2. Stipa gigantea Lag. und St. Lagascae R. et Sch. möchten wohl nur als die robustere, kahlere und die kleinere, behaarte Form derselben Art zu betrachten sein. In den Blüthentheilen fand ich keinen Unterschied, als dass die Deckspelze von St. Lagascae durchschnittlich etwas kürzer ist, als die von St. gigantea, und dass die Granne etwa in demselben Verhältnisse verkürzt erscheint. Die Blătter der St. gigantea sind aussen kahl, am Rande gewimperl, innen fein kurzhaarig; die von St. Lagascae fand ich stets aussen abstehend kurzhaarig, innen mit mikroskopischen Härchen besetzt; die Ligula von St. gigantea ist etwas spitzer und aussen deutlicher behart, die Deckspelze von St. Lagascae hat gegen die Spitze hin zahlreiche Härchen, die beim Abbrechen der Granne als ein Pinselchen vorragen; die von $S t$. gigantea hat deren nur einige wenige. Ausgeprägle Exemplare beider Formen haben einen deutlich verschiedenen Habitus, allein es finden sich bei Vergleichung eines Materials von etwa 50 Exemplaren, wie es mir zu Gebote steht, Mittelformen, so z. B. zeigen Exemplare von St. gigantea bei Escorial gesammelt, die Blätter vom Grunde an bis etwa $1 / 4$ der Länge abstehend behaart. Ich möchte daher die St. Lagascae als forma pubescens der St. gigantea bezeichnen.

Diese Forma pubescens überzieht gesellig wachsend die Gypshügel von Aranjuez, sowie die Hügel am Darro bei Granada; die eigentliche Stipa gigantea hingegen herrscht am Fusse der Sierra Guadarrama, findet sich aber auch auf der Sierra de Alfacar bei Granada.

3. Aristida elatior Cav. sammelte ich bei Malaga im Thale hinter dem Cerro San Anton. Von demselben Standorte sah ich auch ein Exemplar Lagascas im königl. Museal-Herbar zu Madrid. Sie unterscheidet sich von der A. caerulescens Desf., wie sie im Südosten (Valencia, Orihuela, Callosa) vorkommt, durch einen deutlich perennirenden Wurzelstock und eine mehr weitschweifige Rispe, deren 
Aeste sehr verlängert and dünn sind und daher nicken. Die Länge der Hüllspelze gegenüber der Deckspelze ist wechselnd und nicht zur Unterscheidung brauchbar. Ueberhaupt ist diese Pflanze nur die kräftiger vegetirende, ausdauernd gewordene Form der zweijahrigen $\boldsymbol{A}$. caerulescens Desf.

4. Aristella bromoides Bert., von der in Willkomm's Prodromus keine sicheren Standorte angegeben werden, ist häufig am Monte Jabalcuz bei Jaen, an der Wasserleitung der Alhambra bei Granada, an Felsen nachst der Quelle "El Puche" am Camino de los neveros bei Granada. Sie hat im frischen Zustande vollkommen flache Blätter, welche sich jedoch beim Trocknen ganz einrollen, daher wohl die Angabe im Prodromus. Ueberhaupt habe ich sehr viele Pflanzen, denen folia convoluta zugeschrieben werden, bald ausnahmsweise, bald regelmässig mit flachen Blättern gefunden So z. B. Macrochloa arenaria am Puerto de Malagon, die mil ihren breiten, flachen Blättern ganz fremdartig aussieht.

5. Mitium scabrum Merl. beim Escorial ist, wie schon v. Uechtritz in dieser Zeitschrift Jahrg. 1874, pag. 134 vermuthet, besser zu M. IIIontianum Parl. zu zählen, denn es hat eine ausgebreiteto Rispe mit grossen Aehrchen.

6. Molineria minuta Parl. $\beta$. baetica Wk., bei Algeciras häufig, zeichnet sich nicht nur durch höheren Wuchs und gedrängtere Rispe, sondern auch durch die langen Haare, welche am Grunde der Deckspelze entspringen und sie an Länge fast erreichen, vor der Stammform aus.

7. Molineria lendigera möchte ich die Aira lendigera Lagasca zu nennen vorschlagen, sie gehört keineswegs in die Gatlung Aira, denn ihre Hüllspelzen sind bedeutend kürzer als das Aehrchen, die Deckspelze ist an der Spitze abgerundet und unregelmässig klein gezähnelt, was alles mit dem Charakter von Molineria weit besser stimmt als mit Aira. Dazu kommt noch, dass die M. lendigera den grösseren Formen der M. minuta so ähnlich sieht, dass man sie für eine begrannte Varietät derselben hallen könnte. In der That habe ich Exemplare von M. lendigera aus Portugal, von Hofmannsegg gesammelt, gesehen, welche als Aira minuta $\beta$. aristata bezeichne waren. Bei Escorial wachsen beide Arten neben einander, und ma hat daher Gelegenheit, ihre Unterschiede zu studiren.

8. Deschampsia flexuosa $\beta$. stricta Gay. Eine ausgezeichnete Form, deren Rispe selbst zur Blüthezeit schmal lineal ist, da die Aeste hochstens auf $1 / 5$ ihrer Länge nackt sind. Die Aehrchen sind weit grösser als an der gewöhnlichen Form, etwa $8^{\mathrm{mm}}$; ihre Stiele sind (mil Ausnahme derer an den Gipfeln der Zweige) kürzer als das Aehrchen selbst, oft nur $1 / 2$ so lang. Dadurch erscheint die ganze Rispe ungemein gedrungen. Sonst unterscheidet sich diese Form noch durch sehr fein zugespitzle Blälter. Ich sammelte sie auf der Sierra de Cintra in Portugal.

9. Avena fallax R. Sch. (A. montana Vill.?) findet sich in einer Form mit behaarten Blättern auf dem Dornajo in der Nevada, sowie 
auf der Sierra de Alfacar. Diese Form sieht der im trockenen $\mathrm{Zu-}$ stande eingerollten Blätter wegen der A. filifolia Lag. ähnlich, unterscheidet sich jedoch hauptsächlich durch die schmutzig hellbraunen, schlaffen Scheiden der abgestorbenen Blätter, während dieselben bei $A$. filifolia, die ich am Originalstandorte Lagascas (bei Orihuela) sammelte, glänzend zinnoberroth, sehr derb und ganz eng anschliessend sind. Die Blätter der letzteren, auch die halmständigen, sind fest zusammengerollt, auch im lebenden Zustande; bei $A$. fallax sind die Stengelblatter (auch an trockenen Exemplaren) ganz flach, die Blätler der unfruchtbaren Triebe rinnig, und wenn geschlossen, sind sie leicht auszubreiten. Auf der Sierra de Alfacar finden sich beide Arten neben einander. Es scheint, dass die A. filifolia $\beta$. velutina Boiss. Voy. sich vielmehr auf meine $A$. fallax velutina bezieht, wenigstens sprechen dafür die Standortsangaben im Prodromus. Uebrigens bemerke ich noch, dass ich unter den zahlreichen Exemplaren mit abstehender Behaarung der Blätter auch solche mit völlig kahlen gefunden habe, es ist also dieses Merkmal nicht wesentlich.

10. Die ausdauernden Avena-Arten mit verlängerter Ligula, welche in Spanien vorkommen, lassen sich folgendermassen analytisch zusammenstellen:

1. Aehrchenspindel kahl, nur der Callus der Deckspelze mit einem über denselben kaum hervorragenden Haarbüschel; Hüllspelzen weit kürzer als die darüber stehenden Deckspelzen; diese nach oben verbreitert, abgestutzt, ganz oder kurz zweizähnig: . . . . . . . . A. bromoides Gou.

* Die Spindel des Aehrchens behaart, die Haare vom Callus herablaufend; dieser mit einem Büschel von $1 / 10-1 / 6$ der Länge der Deckspelze versehen

2. Deckspelze nach oben allmälig verschmälert, zweizähnig oder -spaltig, die Zähne in lange, feine Haarspitzen auslaufend, gewöhnlich noch von zwei seillichen, sehr kurzen begleitet

* Deckspelze plötzlich zugespitzt, in zwei kurze Zähne ohne Haarspitzen oder in sehr kurze auslaufend . . . . . . 4

3. Deckspelze in ihrer unteren Hälfte kahl, aber längs der Nerven tief gefurcht . . . . . . . A. sulcata Gay.

* Deckspelze in der unteren Hälfte angedrücht seidenhaarig, leicht gefurcht . . . . . . . . A. albinervis Boiss.

4. Deckspelze sehr rauh, an der unteren Hälfte braun, in der Mitte violett, gegen die Spitze weiss gefärbt; obere Hüllspelze kürzer als die über ihr stehende Deckspelze; Rispe kurz, oval . . . . . . . . A. Scheuchzeri All.

* Deckspelze glatt, kaum gefurcht, im unteren Theile grün, sonst weiss; obere Hüllspelze länger als die über ihr stehende Deckspelze; Rispe lang und schmal, oft ährenförmig

5. Rispenäste ganz kahl und glatt, Blätter kurz, breit lineal, mit einwärls gekrümmter Stachelspitze, ganz kahl und glatt, 
mit Ausnahme des fein gezähnelten, sebr dicken und knorpelig-schwieligen weissen Randes . . . . . A. levis mihi.

* Rispenäste durch schaıfe Borstchen rauh; Blätter lang linealisch, zugespitzt, oberseits rath, unterseits erhaben nervig, mit sehr schmalem, weissen kande . . A. pratensis L. Die Blätter aller Arten dieser Gruppe sind mit einem weissen, fein gezähnelten Knorpelrande versehen; am auffallendsten ist derselbe bei $A$. levis, wo er eine dicke Schwiele vorstellt, schwächer, aber noch sehr deutlich bei $A$. bromoides, weniger deutlich ist er bei $A$. albinervis (daher der Name unpassend!), dann bei $A$. sulcata, am schwächsten ist er bei A. pratensis und A. Scheuchzeri.

Avena bromoides scheint durch ganz Spanien verbreitet zu sein; A. sulcata fand ich in grosser Menge auf der Serra de Cintra in Portugal, dann bei Orense und bei Escorial, A. albinervis findet sich massenhaft auf der Sierra de Palma bei Algeciras und dem Monte Carbonera bei San Roque, Boissier's Standort auf der Sierra de Estepona ist demselben benachbart; auf der Sierra Nevada, wo sie nach Willkomm's Prodr. vorkommt, fand ich sie nicht, und dürften die diessbezüglichen Angaben auf $A$. levis m. zurückzuführen sein, wie denn überhaupt die Diagnose von A. albinervis in Willkomm's Prodr. sehr gut zu meiner $A$. levis und nicht zu $A$. albinervis passt, deren hauptsächlichstes von Boissier erwähntes Merkmal, die seidig behaarte Deckspelze, darin nicht angeführt ist. A. Scheuchzeri und $A$. pratensis habe ich nicht selbst in Spanien gesammelt; erstere soll auf den Pyrenäen, letztere in Galicien vorkommen, was vielleicht (nach Willkomm) auf einer Verwechslung mit $A$. bromoides beruht.

11. Avena eriantha Boiss. (sub Arrhenathero) im Süden von Spanien und Portugal sehr verbreitet, ist durch allerlei Zwischenformen mit $A$. elatior $L$. eng verbunden. Die leztere trifft man in Spanien überhaupt nur selten in der Form an, wie sie bei uns vorkommt; die Granne der männlichen Bläthe entspringt meist aus der Basis der Deckspelze, seltener $1 / 5-1 / 4$ der Höhe derselben. Die Deckspelze der Zwitterblüthe ist bald aus der Spitze, bald unterhalb desselben begrannt, bald ist sie kahl, bald mit zerstreuten Haaren besetzt; werden diese etwas dichter, so verschwindet die Grenze gegen $A$. eriantha vollständig.

12. Avena Thorei Duby, wie sie in Galicien und Nord-Portugal häufig vorkommt, hat im lebenden Zustande immer flache Blatter und eine ausgebreitete Rispe, nicht wie in Willkomm's Prodromus steht: "folia basilaria convoluto-filiformia" und "panicula contracta." Letztere Ausdrücke können höchstens bei getrockneten Exemplaren gebraucht werden.

13. Trisetum scabriusculum Coss. bildet in auffallender Weise den Uebergang von Trisetum zur Koeleria und sollte vielmehr letzterer Galtung beigezählt werden. Es ist kaum glaublich, welche grosse Verschiedenheiten die Stellung und Länge der Granne der Dechspelze bei diesem Grase zeigt. Bei typischen Exemplaren entspringt die Granne im oberen Viertel des Rückens der Deckspelze 
und ist etwas kürzer als diese selbst. Dann findet man Exemplare, wo die Granne auf $1 / 6$, endlich auf $1 / 8$ der Länge hinaufrủckt, bis sie endlich knapp unter der Spitze steht und nur noch von 2 häutigen Zähnchen begleitet ist, ganz wie bei Koeleria phleoides und noch mehr bei $K$. macilenta DC. Dabei durchläuft sie alle Längenverhältnisse von dem erstgenannten bis zum vollständigen Schwinden, welches gar nicht selten vorkommt. Auch ist die Granne niemals wie bei anderen Trisetum-Arten gekniet, sondern ganz gerade. Ich möchte daher diese Art künftighin Koeleria scabriuscula Lag. (sub Avena) nennen und sie neben $K$. macilenta und $K$. phleoides stellen. Die grosse Variabilität dieser Art zeigt sich übrigens auch in dem Haarüberzug der Spelzen; meist fehlt derselbe vollständig, häufig aber ist er kurz-flaumig, seltener zottig rauh; zwischen allen diesen Formen existiren übrigens die leisesten Uebergänge.

14. Trisetum velutinum Boiss. hat in der Regel "folia dense velulina albida"; es finden sich aber auch Exemplare mit zerstreutem, kurzen Flaume der lebhaft grünen Blätter, fast wie bei den behaarten Formen des Tris. flavescens, dem sie dadurch sehr ähnlich werden; doch sind sie sogleich durch die Hüllspelzen von einander zu unlerscheiden, welche bei T. velutinum gleichlang sind, während bei Trisetum flavescens die untere nur halb so lang ist als die obere.

15. Holcus lanatus L. Sowohl die spanischen als die portugiesischen Exemplare weichen von den mitteleuropäischen dadurch $a b$, dass die Deckspelze am Grunde mit einigen wenigen aber langen, aus dem Callus entspringenden Haaren besetzt ist. Auch sind die Hüllspelzen über die ganze Oberfläche sehr rauh. Bei einer Form von Puerto de Despeñaperros sind die Scheiden nicht wie bei der normalen "molliter pubescentes", sondern breviter retrorso-hispidae.

16. Holcus annuus Slzm. (setiglunis Boiss.) hat normal Hüllspelzen, deren Grannen ebenso lang sind, als jene selbst; bei einer Form "brevisetus" hingegen (von Puerto de Despeñaperros) beträgt die Länge der Granne nur $1 / 5$ der Hüllspelze.

17. Koeleria crassipes Lge. Es scheint mir wahrscheinlich, dass diese Art synonym ist mit Airochloa caudata Link in Linnaea XVI, p. 405. Die Beschreibung stimmt vollständig mit den bei Escorial gesammelten Exemplaren. Link sammelte sie übrigens in Portugal. Seine Bezeichnung hätte die Priorităt.

Koeleria crassipes $\beta$. nevadensis $\mathrm{m}$. Blätter kurz, rückwärts gekrümmt, sehr lang gewimpert und mit dickem, weissem Knorpelrande. Rispe sehr gedrungen, länglich oval. In der alpinen Region der Nevada beim Boreguil de San Geronimo.

18. Danthonia decumbens DC. sammelte ich in 2 Formen:

a. breviglumis. Hüllspelzen von der Länge des Aehrchens oder etwas kürzer (Sierra de Palma bei Algeciras).

$\beta$. longiglumis. Hüllspelzen um die Hälfte länger als das Aehrchen (Serra de Cintra in Portugal). 
19. Vulpia sciuroides Gmel. var. microstachya: Aehrchen sehr dicht gedrängt, $6 \mathrm{Mm}$. lang; untere Hüllspelze 2, obere $5 \mathrm{Mm}$., Deckspelze $6 \mathrm{Mm}$., sehr schmal lineal, ihre Granne $8 \mathrm{Mm}$. Bei San Roque.

20. Festuca Clementei Boiss. Eine vortrefflich charakterisirte Art, welche durch ihre in horizontale Fasern aufgelösten alten Blattscheiden sehr ausgezeichnet ist. Der Halm ist stets blattlos. Am Picacho de Veleta in der Sierra Nevada.

21. Festuca duriuscula var. effusa. Rispenäste sehr lang und dünn, nur an der Spitze mit Aehrchen versehen, weit abstehend, kahl und glatt. Puerto de Despeñaperros; S. de Alfacar.

22. Festuca rivularis Boiss. Ist keine Varietät von F. durinscula, wo sie im Prodr. Fl. hisp. untergebracht ist; eher gehört sie zu $F$. rubra. Thre grundständigen Blätter sind gefalzt, gekielt, sehr platt gedrückt, fast schwertförmig, ganz glatt, selbst am Rande. Halmblätter vollständig flach, Ligula $\theta$. Rispe sehr locker, die langen Aeste erst an ihrem Ende ährchentragend. Aehrchen lang, Deckspelze deutlich 5nervig. Im obersten Jenil-Thale.

23. Festuca caerulescens Desf. Die alten Scheiden sind in Fasern aufgelöst, der Halm ist niedrig, die Blätter alle flach, mit kaum merklicher Ligula, die Rispe sehr schmal. Sierra de Palma bei Algeciras, Monte Carbonera bei San Roque.

24. Festuca spadicea L. Der vorigen nahe verwandt, hat ganze, unzerfaserte alte Scheiden, der Halm ist hochwüchsig, die Rispe oval; die grundständigen Blätter sind oft eingerollt, die Halmblätter flach, ihre Ligula lang vorgezogen. In grösster Menge auf Serra de Cintra und Serra de Bussaco in Portugal, dann bei Orense in Galicien und Villafranca del Vierzo in Leon, endlich bei Peñones San Francisco auf der S. Nevada. ( $F$. caerulescens, welche im Prodromus daselbst angegeben ist, habe ich dort nicht gefunden.)

25. Festuca altissima Boiss., welche ich in einer feuchten Bachschlucht der $\mathbf{S}$. de Palma bei Algeciras fand, ist mit unserer niederösterr. F. Drymeja M. et K. absolut identisch.

26. Brachypodium mucronatum $\mathrm{Wk}$. Eine Form mit behaarten Aehrchen, sehr stumpfer Deckspelze mit kaum wahrnehmbaren Stachelspitzchen sammelte ich bei Villanova de Portimão in Portugal.

27. Desmazeria loliacea Nym. Bei Malaga und Algeciras kommen Formen mit verästelter Rispe vor.

28. Lolium perenne L. auriculatum. Eine Form mit sehr langen, weit übergreifenden Oehrchen des Blaltgrundes. In Wäldern bei Huéjar de Sierra am Jenil.

Zum Schlusse noch einige interessantere Standorte von in Spanien und Portugal wenig verbreiteten Arten:

Anthoxanthum ovatum Lag. Sehr häufig in den Umgebungen von Algeciras und San Roque.

Alopecurus castellanus Boiss. et Reut. Auf Wiesen bei Villafranca del Vierzo in Leon. 
Andropogon distachyon L. Bei AJgeciras, San Roque.

Agrostia canina var. $\gamma$. humilis $\mathbf{W} \mathrm{k}$. Borreguil de San Juan in der S. Nevada.

- castellana Boiss. et Reut. Puerto de Despeñaperros.

- nebulosa Boiss. el Reut. Um Granada sehr verbreitet: im Darrothale, bei El Puche am Camino de los neveros, selbst in der Alameda del Jenil.

- Reuteri Boiss. Im Darrothal bei Granada und bei Loja.

- pallida DC. Sehr häufig im Puerto de Despeñaperros.

Chaeturus fasciculatus Lk. S. de Cintra.

Airopsis globosa Desv. Bei Orense in Galicien auf allen trockenen Hügeln; ferner in der S. de Cintra in Portugal.

Corynephorus articulatus P. B. Sandige Orte bei Sines in Portugal. Deschampsia media R. S. Auf feuchten Weiden bei El Puche nächst Granada.

Avena longiglumis Dur. Auf Sandplätzen bei Sines in Portugal. - strigosa v. uniflora m.! Auf Feldern bei Bussaco in Portugal.

Holcus glaucus Wk. An allen feuchten Stellen um Granada bis in die subalpine Region hinauf.

- Gayanus Boiss. In Felsspalten bei Villafranca del Vierzo in Leon. Koeleria macilenta DC. Bei Almeria, am Cerro San Anton bei Malaga, dann bei Portimão und Cintra in Portugal.

Melica major S. et S. In Galicien bei Orense.

- Magnolii Gren. et Godr. Am M. Santo bei Lissabon.

Sphenopus Gouani Trin. An salzigen Stellen beim Cabo de Gata.

Bromus molliformis Lloyd. An Ackerrändern bei Malaga.

- humilis Cav. An Rainen bei Algeciras.

- macrostachys Desf. Ueberall bei Algeciras und San Roque, Villanova de Portimăo.

- asper L. In Hainen bei der Alhambra.

Aegilops ventricosa Tausch. Puerto de Despeñaperros.

St. Pölten, den 10. Februar 1877.

Nachschrift. Unter den von meinem Reisegefährten, Herrn Winkler gesammelten und mir zur Revision übergebenen Gräsern befindet sich auch die bisher nur aus Sizilien bekannte Agrostis nitida Guss., welche Winkler in 2 Individuen am Cabo de Gata bei Almeria sammelte, wo sie jedenfalls selten zu sein scheint, da ich selbst sie übersehen habe. 